\title{
Discussion on the Application of Enterprise Business Intelligence Data Analysis System
}

\author{
Ning Yang* \\ Weifang Special Steel Group Co., Ltd., Weifang 261200, Shandong Province, China
}

*Corresponding author: Ning Yang, yang6168@139.com

\begin{abstract}
Enterprise Business Intelligence (BI) system refers to data mining through the existing database of the enterprise, and data analysis according to customer requirements through comprehensive processing. The data analysis efficiency is high and the operation is convenient. This paper mainly analyzes the application of enterprise BI data analysis system in enterprises.
\end{abstract}

Keywords: Enterprise; BI data analysis system; Application

Publication date: July 2021; Online publication: July 31, 2021

\section{Introduction}

In the era of data, the current development of enterprises has a large amount of data information that will be generated in various links such as procurement, production, operation and sales. This plays an important guiding role in the development of enterprises. Through the application of information technology, the massive historical data are integrated and analyzed. This is to summarize the experience and lessons in each link of the enterprise, as well as use the data to guide the decision-making and operation mode of the enterprise, and predict the future development of the enterprise, which is conducive to promoting the better development of the enterprise. BI data analysis system is the key to data processing in all links of enterprises. It is more and more frequently used in enterprise data analysis, and can form an index analysis system through data analysis to provide corresponding reference for enterprise development decision-making.

\section{Project content on the application of enterprise BI data analysis system}

With the development of the enterprise, the business items and business scale of the enterprise will gradually increase. At the same time, the production, operation and sales outlets of the enterprise are also expanding. The enterprise needs to combine a large amount of data generated in each link to build an information database system to make statistics on the data, so as to meet the needs of the development of production and operation of the enterprise. However, with the increase of data, the traditional Enterprise Resource Planning (ERP), Point of Scale (POS) and other systems of enterprises have been unable to meet the production requirements of enterprises, and the decision-making information provided for enterprises is also poorer, making it more difficult to transform and integrate data ${ }^{[1]}$. In addition, with the increase of databases, the phenomenon of heterogeneous environment is obvious. And how to do a good job in heterogeneous environment of data conversion is particularly necessary. The business of the enterprise is relatively scattered and the number of databases is increasing. A platform that can integrate various data of the enterprise is needed to unify the analysis of enterprise reports and integrate the business status of the enterprise. Through the analysis of core indicators, the horizontal and vertical comparison of data is realized, so as to provide effective strategies for the operation and development of enterprises. 


\section{Construction objectives of project data analysis system}

In the construction of the project data analysis system, Extract-Transform-Load (ETL), Oracle and other joint applications are used to design a BI analysis management platform, sort out and analyze the business indicators, and achieve the following objectives:

(1) First, build a network business intelligence platform, integrate and transmit different database data, realize data sharing and solve the problem of isomerization data.

(2) Second, the system can generate business analysis reports to improve the efficiency of enterprise data analysis.

(3) Third, the data business of the project should be comprehensively and multi-dimensionally analyzed.

(4) Fourth, diversified presentation of the statement interface to make the form of the statement more diverse.

(5) Fifth, expand the functions of the system and connect with other businesses effectively.

(6) Sixth, streamline the operation of reports, so that the definition, distribution and maintenance of reports have standardized processes.

\section{Architecture of BI data analysis system}

BI data analysis system includes source data layer, data extraction layer, data staging layer, data warehouse layer and data application layer. Among them, the source data layer mainly analyzes the source data in the enterprise. The data generated by various businesses in the daily operation and management of an enterprise can be used as source data, including purchase data, business transaction data, sales data and financial data. The data extraction layer is the extraction of source data in enterprise business ${ }^{[2]}$. Through the extraction of source data in the data extraction layer and data analysis, it provides a reference for future data research and verification. The data staging layer refers to the operation storage area of data, including data extraction, conversion, storage and other processing contents, so as to provide support for data warehouse source data analysis. The data warehouse layer is a form of operation using the staging layer, which provides query services for users through calculation, processing and data warehouse storage; In the architecture of the application layer, the access business is carried out through the Browser/Server Architecture. It can generate various work and analysis reports, which is conducive to the analysis of enterprise procurement, production, sales and finance, optimize enterprise resource allocation and improve enterprise user management efficiency.

\section{Influence factors and solutions of BI data analysis system}

\subsection{Incomplete capture of Manufacturing Execution System (MES) scanning points}

Through the application of BI data analysis system, there are still imperfect problems in the capture of MES scanning points. For example, in the data analysis of product repair qualification rate in the BI data analysis system, the program setting of the system requires that the repaired goods can be captured by the BI system only after they are online, frozen and qualified for repair through scanning of each program. However, if the product is repaired more than twice, there will be differences with the set data logic in the scanning of these three links, and the BI system will miss grasping in the grasping of repaired qualified products, resulting in inaccurate data collection of product repair qualification rate. Therefore, in the design of the system, it is necessary to do a good job in information capture logic revision to integrate heterogeneous data and ensure that similar data in the system can be captured accurately. Through many tests, the captured data of BI data system is compared with manual data to ensure the accuracy of logic formulation.

\subsection{The manual data upload of $B I$ system is unreasonable}

In BI system, some data can be captured directly by the system, and some data need to be input by manual 
data. For example, for customer complaint information entry, the data information entry specialist needs to download the standard template on the BI data platform, and then upload the data to the data collection platform ${ }^{[3]}$. However, if the data filling format is wrong in the process of uploading, or the data is uploaded before the format is set, it is easy to cause the BI data analysis system platform to be unrecognized. For example, if the date format is set to $20 \mathrm{XX} / \mathrm{XX} / \mathrm{XX}$, but it is entered as XX / XX / 20XX during the input process, the system will not recognize it. To solve this problem, the uniformity must be ensured in the design of the data acquisition platform template. The input data must be consistent with the requirements of the template, otherwise it cannot be submitted, or the system will automatically give a prompt to ensure the accuracy and effectiveness of manual information input through the setting of error prevention function.

\subsection{Time differences existed in data synchronization}

In the application of BI data analysis system, the data capture time needs to be set. The usual setting time is 8:00 a.m. to 8:00 a.m. the next day. However, there will be time error in the system. MES or System Applications and Products (SAP) data capture was not carried out at exactly 8:00, resulting in incomplete data. Aimed at this problem, due to the time of error only about 1 minute around 8:00, the error is not big. The enterprise can combine business situation, compare the data capture situation of the system with manual recording data, view the difference between the two, determine how to improve, or do not make system improvement through business adjustment.

\subsection{System processing lag}

In the data processing of BI data analysis system, Quality Management System (QMS) / MES and other modules are prone to lag, resulting in deviation of BI system indicators. For example, in the calculation of quality loss, the QMS system has given unqualified documents, but did not give reasonable treatment opinions, or in the statistics of unqualified products in the MES system, the products have been frozen without giving accurate judgment results. In the absence of an evaluation, the BI data analysis system cannot accurately capture the data. As a result, the quality loss index is not fully reflected and the index value of the current day is inaccurate. After the processing of unqualified documents is completed on the second day, the product will be judged as scrap, and the quality loss will be regarded as a numerator, but the loss of the day cannot be accurately expressed, affecting the index value. To solve this problem, enterprises can require to make handling opinions within 24 hours in system design and management. If it is not handled in time, the process will jump automatically. Although the indicators of the current day may be affected, as long as the statistical process of nonconforming products can be completed in the current month, the quality loss value within one month will not be affected.

\subsection{The handover data is inconsistent}

Some enterprises will adopt the two-shift system in the application of BI data analysis system, so that there are great differences between handover data, which can be analyzed from two aspects. The first is that the personnel on duty do not process the QMS system from the completion of the task of the shift to the time of taking over the shift, so that the accumulation of these data will enter the work of the next team, which may lead to inaccurate data processing because the next team does not participate in the work. Or the BI system has time differences in the setting of shift system. For example, the system setting requires that the first shift information capture time is 8:00 a.m. to 8:00 p.m. The information capture time of the second team is from 8:00 p.m. to 8:00 a.m. the next day. However, the actual working time of the team may be advanced or delayed by about 10 minutes, resulting in inaccurate data capture and chaotic data recording of the two teams. In view of these two problems, it can be required that each team must complete their own work before shift handover. The work of this shift cannot be left to the next team. At the same time, no 
operation will be carried out 10 minutes before shift handover.

\section{Attention issues of $B I$ data analysis system application \\ 6.1. Support from leaders}

The application of BI data analysis system is inseparable from the support of leaders, which is strictly implemented from top to bottom. At the same time, enterprise leaders need to authorize the corresponding person in charge to ensure the scientific operation of the system. At the same time, in the system import, leaders need to take the lead to standardize the use of the system, and put forward the corresponding improvement and improvement strategies combined with their own applications.

\subsection{Sort out business indicators}

Sorting out business indicators in BI data analysis system is the basis of system application. Logical compilation can be completed through sorting out indicators, so as to better guide the docking and development of business systems and ensure the accuracy of data checking. In combing, the logic and operability of the indicators should be analyzed to ensure the rationality of the formulation of the indicators.

\section{Conclusion}

To sum up, the BI data analysis system provides a good data integration and analysis platform for the enterprise, and provides accurate statistical analysis reports for the work of each business department of the enterprise. Moreover, it can provide comprehensive and scientific decision-making data information for enterprises through the analysis of statements, so that enterprises can optimize enterprise development objectives and business means by adjusting operation strategies.

\section{Disclosure statement}

The author declares no conflict of interest.

\section{References}

[1] Li WW. 2017, Application of Enterprise BI data Analysis System. Computer Knowledge and Technology, 13(24): 11-12,14.

[2] Song X, Guo J, Yin SY, et al., 2015, Application of Business Intelligence in Data Analysis of Power Grid Dispatching Control System. Automation of Electric Power Systems, 39(12): 93-96, 145.0/AEPS20140811007.

[3] Yang JY, 2018, Design and Implementation of Educational Intelligence Analysis System based on BI Technology. University of Chinese Academy of Sciences. 